\title{
Magnetic Domain Structure and Magnetic Hardening in Sm-Fe-Mn-N Coarse Powder
}

\author{
J. J. Kim ${ }^{1}$, H. S. Park ${ }^{1}$, D. Shindo ${ }^{1, *}$, T. Iseki ${ }^{2}$, N. Oshimura ${ }^{2}$, T. Ishikawa ${ }^{2}$ and K. Ohmori ${ }^{2}$ \\ ${ }^{1}$ Institute of Multidisciplinary Research for Advanced Materials, Tohoku University, Sendai 980-8577, Japan \\ ${ }^{2}$ Ichikawa Research Laboratory, Sumitomo Metal Mining Co., Ltd., Chiba 272-8588, Japan
}

\begin{abstract}
The magnetic domain structure and magnetization process in $\mathrm{Sm}_{2}\left(\mathrm{Fe}_{0.95}, \mathrm{Mn}_{0.05}\right){ }_{17} \mathrm{~N}_{5.0}$ powders are investigated by Lorentz microscopy and electron holography. The magnetic domain walls are aligned along the magnetization easy axis (c axis) and are connected with one another along amorphous layers that are distributed along the direction parallel to the $\mathrm{c}$ axis. On the other hand, it is found that the domain walls are always located in the amorphous layer after domain wall motion. These results reveal that the amorphous layers are the pinning centers of the domain walls. Thus, it is reasonable to consider that the coercivity of a $\mathrm{Sm}_{2}\left(\mathrm{Fe}_{0.95}, \mathrm{Mn}_{0.05}\right)_{17} \mathrm{~N}_{5.0}$ powder is strongly affected by the distribution of the amorphous layers in the powder. [doi:10.2320/matertrans.MD200702]
\end{abstract}

(Received April 3, 2007; Accepted May 1, 2007; Published July 11, 2007)

Keywords: Samarium-iron-manganese-nitrogen coarse powder, coercivity mechanism, Lorentz microscopy, electron holography

\section{Introduction}

$\mathrm{Sm}_{2} \mathrm{Fe}_{17} \mathrm{~N}_{\delta}$ fine powders have attracted considerable attention as high-performance permanent magnets for their high saturation magnetizations and for their strong uniaxial anisotropies that are comparable to those of $\mathrm{Nd}_{2} \mathrm{Fe}_{14} \mathrm{~B}$ permanent magnets. ${ }^{1-4)}$ In particular, thus far, a $\mathrm{Sm}_{2} \mathrm{Fe}_{17} \mathrm{~N}_{3}$ fine powders with an average size of $2 \sim 3 \mu \mathrm{m}$ have been used as bonded magnets. However, fine powders suffer from the problems of poor oxidation resistance and thermal instability of coercivity; furthermore, a high compaction pressure is required for the densification of these powders in order to obtain high-energy products in various applications. Hence, several studies have attempted to obtain high coercivity in coarse powders by adding elements such as Ta, Co, W, and $\mathrm{Mn}$ to $\mathrm{Sm}_{2} \mathrm{Fe}_{17} \mathrm{~N}_{\delta}$ alloys. ${ }^{4-7)}$ Recently, $\mathrm{Sm}_{2}(\mathrm{Fe}, \mathrm{Mn})_{17} \mathrm{~N}_{\delta}$ coarse powders with average sizes of $20 \sim 30 \mu \mathrm{m}$ have been developed by Iseki et al.;) these powders exhibit $(\mathrm{BH})_{\max }$ values of greater than $175 \mathrm{~kJ} / \mathrm{m}^{3}$, good oxidation resistance, and high coercivity $(1.04 \mathrm{MA} / \mathrm{m})$.

It is well known that the magnetization reversal mechanism that determines the coercivity of a $\mathrm{Sm}_{2} \mathrm{Fe}_{17} \mathrm{~N}_{\delta}$ fine powder is controlled by nucleation. ${ }^{9)}$ On the other hand, the coercivity mechanism in $\mathrm{Sm}_{2}(\mathrm{Fe}, \mathrm{Mn})_{17} \mathrm{~N}_{\delta}$ coarse powders remains unclear at present, although it is considered to be controlled by pinning. Recently, we have reported that in a $\mathrm{Sm}_{2}\left(\mathrm{Fe}_{0.95}, \mathrm{Mn}_{0.05}\right){ }_{17} \mathrm{~N}_{4.2}$ coarse power, the amorphous phase is enriched with $\mathrm{N}$ and $\mathrm{Mn}$, while the crystalline phase is enriched with Fe. Furthermore, it has been clarified that a domain wall exists in Mn-enriched amorphous phases. Some preliminary experimental results have been published in a previous study. ${ }^{10)}$ In this study, the magnetic domain structure in $\mathrm{Sm}_{2}\left(\mathrm{Fe}_{0.95}, \mathrm{Mn}_{0.05}\right)_{17} \mathrm{~N}_{5.0}$ coarse powders is investigated in detail by Lorentz microscopy. Further, in order to study magnetic hardening in these powders, ex situ observations are carried out by Lorentz microscopy and electron holography.

*Corresponding author, E-mail: shindo@tagen.tohoku.ac.jp

\section{Experimental Procedure}

In this study, $\mathrm{Sm}_{2}\left(\mathrm{Fe}_{0.95}, \mathrm{Mn}_{0.05}\right){ }_{17} \mathrm{~N}_{5.0}$ coarse powders were prepared by the nitridation of SmFeMn obtained by a reduction and diffusion (RD) process. ${ }^{8)}$ For transmission electron microscopy observations, thin foil specimens were prepared by a focused ion beam (FIB) method (FB-2000A, FB-2100) using a microsampling technique. Ex situ observations were carried by Lorentz microscopy and electron holography using a JEM-3000F transmission electron microscope (TEM), in which a field emission gun and biprism were installed. The residual magnetic field around the specimen position in the TEM was reduced to around $32 \mathrm{~A} / \mathrm{m}$ by switching off and then degaussing the objective lens. ${ }^{11)}$ Electron holograms were recorded on conventional films and digitized using a film scanner having a resolution of $8 \mu \mathrm{m} /$ pixel. Reconstructed phase images of the electron holograms were obtained using a Fourier transform for the digitized holograms. Ex situ experiments were carried out by using an electromagnet that was designed to apply a magnetic field of up to $2.5 \mathrm{~T}$ to the thin foil specimen placed in the TEM holder. In these experiments, the thin foil specimen to be observed was removed from the TEM and subjected to a strong magnetic field. The remanent state of the specimen was then observed by inserting the specimen back into the TEM.

\section{Results and Discussion}

Figure 1 shows the bright-field, dark-field, and Lorentz microscope images of the $\mathrm{Sm}_{2}\left(\mathrm{Fe}_{0.95}, \mathrm{Mn}_{0.05}\right)_{17} \mathrm{~N}_{5.0}$ powder. These images were obtained from the [1 10$]$ zone axis, where the specimen includes the $\mathrm{c}$ plane, i.e., the magnetization easy axis (c axis) is located in the specimen plane. Lorentz microscopy is performed using the Fresnel mode under the defocused condition, in which the white and black lines correspond to the magnetic domain walls. ${ }^{12)}$ In Fig. 1(c), it is found that the magnetic domain walls are almost parallel to the $\mathrm{c}$ axis. On the other hand, in Fig. 1(b), which shows the dark-field image taken using 006 reflection, lined shapes of a 


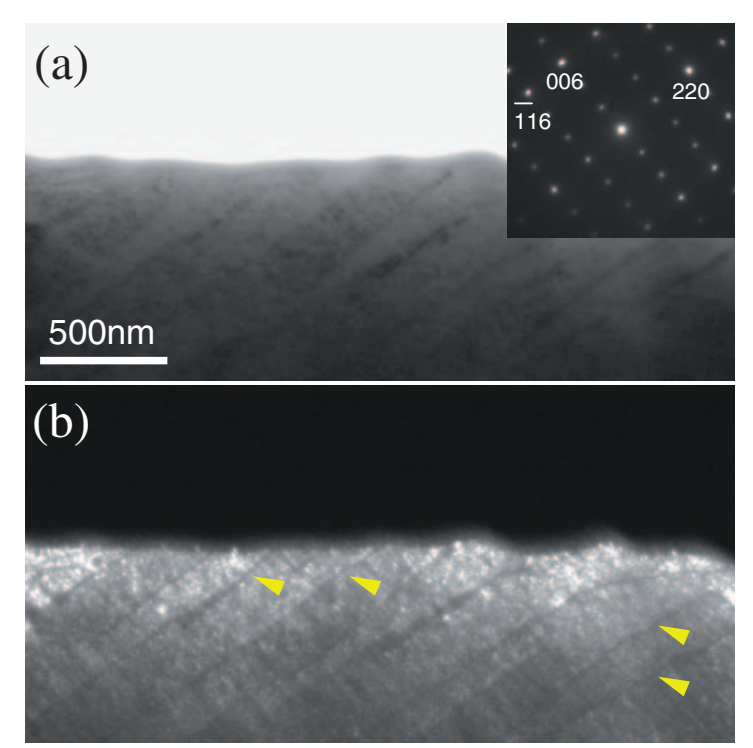

(c)

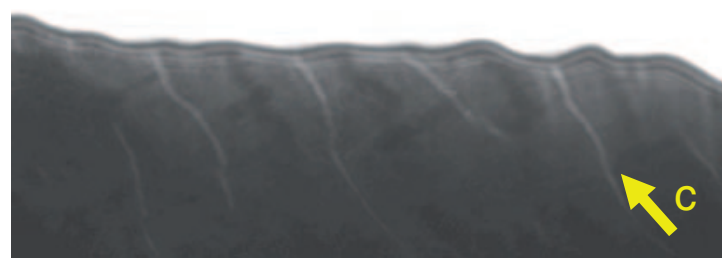

Fig. 1 (a) Bright-field image and (b) dark-field image of the $\mathrm{Sm}_{2}\left(\mathrm{Fe}_{0.95}, \mathrm{Mn}_{0.05}\right)_{17} \mathrm{~N}_{5.0}$ powder. The inset of (a) shows a diffraction pattern. (c) Corresponding Lorentz microscope image taken in the underfocused condition. The magnetization easy axis is indicated by a yellow arrow.

dark contrast (indicated by yellow arrowheads), which are almost perpendicular to the c axis, are clearly observed. This result implies the existence of another phase within a crystallized grain of the $\mathrm{Sm}_{2}\left(\mathrm{Fe}_{0.95}, \mathrm{Mn}_{0.05}\right)_{17} \mathrm{~N}_{5.0}$ powder.

Figure 2 shows the TEM results obtained for another $\mathrm{Sm}_{2}\left(\mathrm{Fe}_{0.95}, \mathrm{Mn}_{0.05}\right){ }_{17} \mathrm{~N}_{5.0}$ powder. In Fig. 2(a), amorphous phases (indicated by white arrows) similar to those in a $\mathrm{Sm}_{2}\left(\mathrm{Fe}_{0.95}, \mathrm{Mn}_{0.05}\right)_{17} \mathrm{~N}_{4.2}$ powder are found. ${ }^{10)}$ Further, the corresponding Lorentz microscope image shown in Fig. 2(b) confirms that the domain walls are located in the amorphous phases. These results are consistent with those of a previous study. ${ }^{10)}$ On the other hand, as shown in Fig. 2(c), it is more clearly seen that the amorphous phases (dark contrast except the parts indicated by yellow arrowheads) are almost parallel to the $\mathrm{c}$ axis in the same manner as the domain walls. Here, it is noted that layered dark-contrast regions indicated by yellow arrowheads are shown in Fig. 2(c). The enlarged image of a dark-contrast region is shown in Fig. 2(d). It is considered that these dark-contrast regions are the same as the lined shapes of the dark contrast observed in Fig. 1(b). From Fig. 2(d), it is found that an amorphous layer (indicated by a white arrow) is connected with another amorphous layer existing inside the layered dark-contrast region and that these layers are almost parallel to the $\mathrm{c}$ axis, as mentioned earlier. In fact, the magnetization process and domain structures in the $\mathrm{Sm}_{2}\left(\mathrm{Fe}_{0.95}, \mathrm{Mn}_{0.05}\right)_{17} \mathrm{~N}_{5.0}$ powder are related to these amorphous layers, as discussed in detail below. Therefore, in the future, we plan to carry out detailed studies on the origins of these layered regions and the effects of the regions on the domain structures and magnetic properties of the $\mathrm{Sm}_{2}\left(\mathrm{Fe}_{0.95}, \mathrm{Mn}_{0.05}\right){ }_{17} \mathrm{~N}_{5.0}$ powder.

On the other hand, in order to observe the shape and distribution of the domain walls, we have carried out Lorentz microscopy experiments based on changes in the tilting angle of the specimen. The tilting axes (x,y) are indicated in Fig. 3(a). Lorentz microscope images are taken in the underfocused condition. As shown in Figs. 3(b)-(f), it is found that a magnetic domain wall links up with another magnetic domain wall and that these domain walls exhibit a lined shape along the $\mathrm{c}$ axis, although the specimen is tilted. Figure 4 shows a Lorentz microscope image taken under the condition of severe underfocus. In the enlarged Lorentz microscope image shown in Fig. 4(b), interference contrast due to the existence of the domain wall is observed within a white line that indicates the domain wall. Furthermore, it is clearly seen that the width of a black band increases with the specimen thickness toward the bottom of the specimen. From these results, it is reasonable to consider that the domain wall is located in the region from the top surface to the bottom surface of the specimen having a thickness of approximately $100 \mathrm{~nm}$ and is tilted toward the incident beam direction.

In order to clarify the magnetic hardening mechanism in the $\mathrm{Sm}_{2}\left(\mathrm{Fe}_{0.95}, \mathrm{Mn}_{0.05}\right){ }_{17} \mathrm{~N}_{5.0}$ powder, ex situ observations of the magnetization process are carried out by Lorentz microscopy. Figure 5 shows the magnetization process in the $\mathrm{Sm}_{2}\left(\mathrm{Fe}_{0.95}, \mathrm{Mn}_{0.05}\right)_{17} \mathrm{~N}_{5.0}$ powder. It is noted that in the Lorentz microscope images, the white band existing from the right-top region to the center-bottom region arises due to the variation in the specimen thickness; this variation is brought about by the use of the FIB milling process. First, a strong magnetic field of $2.5 \mathrm{~T}$ is applied to this specimen using an electromagnet, as shown in Fig. 5(a). In this condition, domain walls are not observed due to the application of the strong external magnetic field of $2.5 \mathrm{~T}-\mathrm{a}$ value that is comparable to the coercivity of the $\mathrm{Sm}_{2}\left(\mathrm{Fe}_{0.95}, \mathrm{Mn}_{0.05}\right){ }_{17} \mathrm{~N}_{5.0}$ powder. Thus, it is considered that the direction of magnetization is the same throughout the powder and that it is parallel to the direction of the applied external magnetic field. In Figs. 5(b) and (c), the domain walls are observed by applying the external magnetic field along the opposite direction. Further, it is found that with an increase in the external magnetic field from $0.5 \mathrm{~T}$ to $1.0 \mathrm{~T}$, the area of the domains magnetized along the direction parallel to that of the external magnetic field increases within the field of view. Figure 6 shows the enlarged Lorentz microscope images obtained for the rectangles indicated by broken yellow lines in Figs. 5(b) and (c). In the just-focused images shown in Figs. 6(a) and (c), the black contrasts indicated by the yellow arrows represent amorphous layers in the same manner as in Fig. 2(a). In the corresponding underfocused images shown in Figs. 6(b) and (d), it can be clearly seen that the domain walls are also located in these amorphous layers after domain wall motion. Consequently, these results reveal that the amorphous layer acts as an attractive pinning center for domain wall motion, resulting in the large coercivity $(1.04 \mathrm{MA} / \mathrm{m})$ of the $\mathrm{Sm}_{2}\left(\mathrm{Fe}_{0.95}, \mathrm{Mn}_{0.05}\right)_{17} \mathrm{~N}_{5.0}$ powder. 

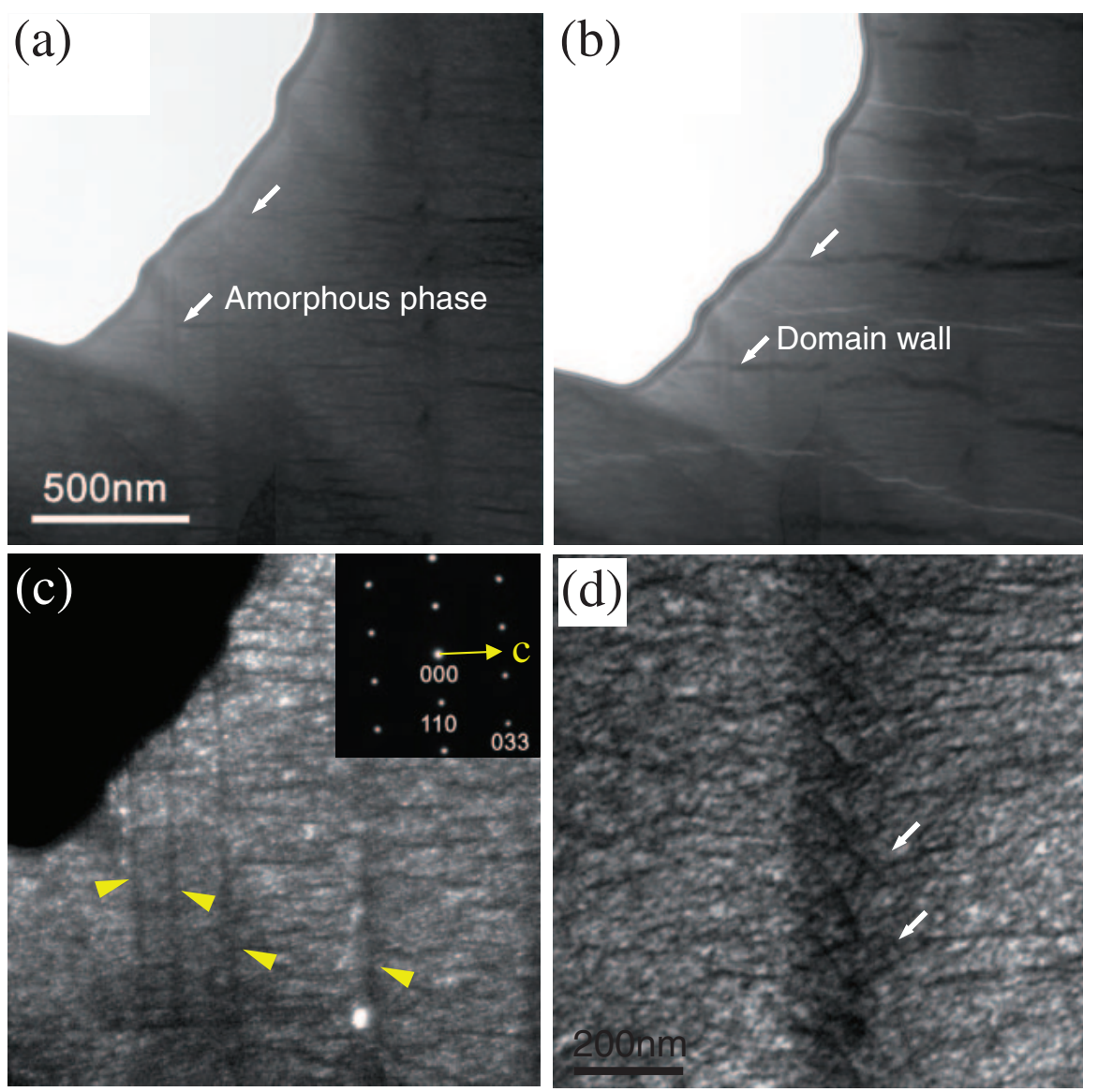

Fig. 2 Lorentz microscope images of the $\mathrm{Sm}_{2}\left(\mathrm{Fe}_{0.95}, \mathrm{Mn}_{0.05}\right)_{17} \mathrm{~N}_{5.0}$ powder taken in the (a) just-focused and (b) underfocused conditions. (c) Dark-field image and diffraction pattern (inset). The magnetization easy axis is indicated by a yellow arrow. (d) Enlarged bright-field image.
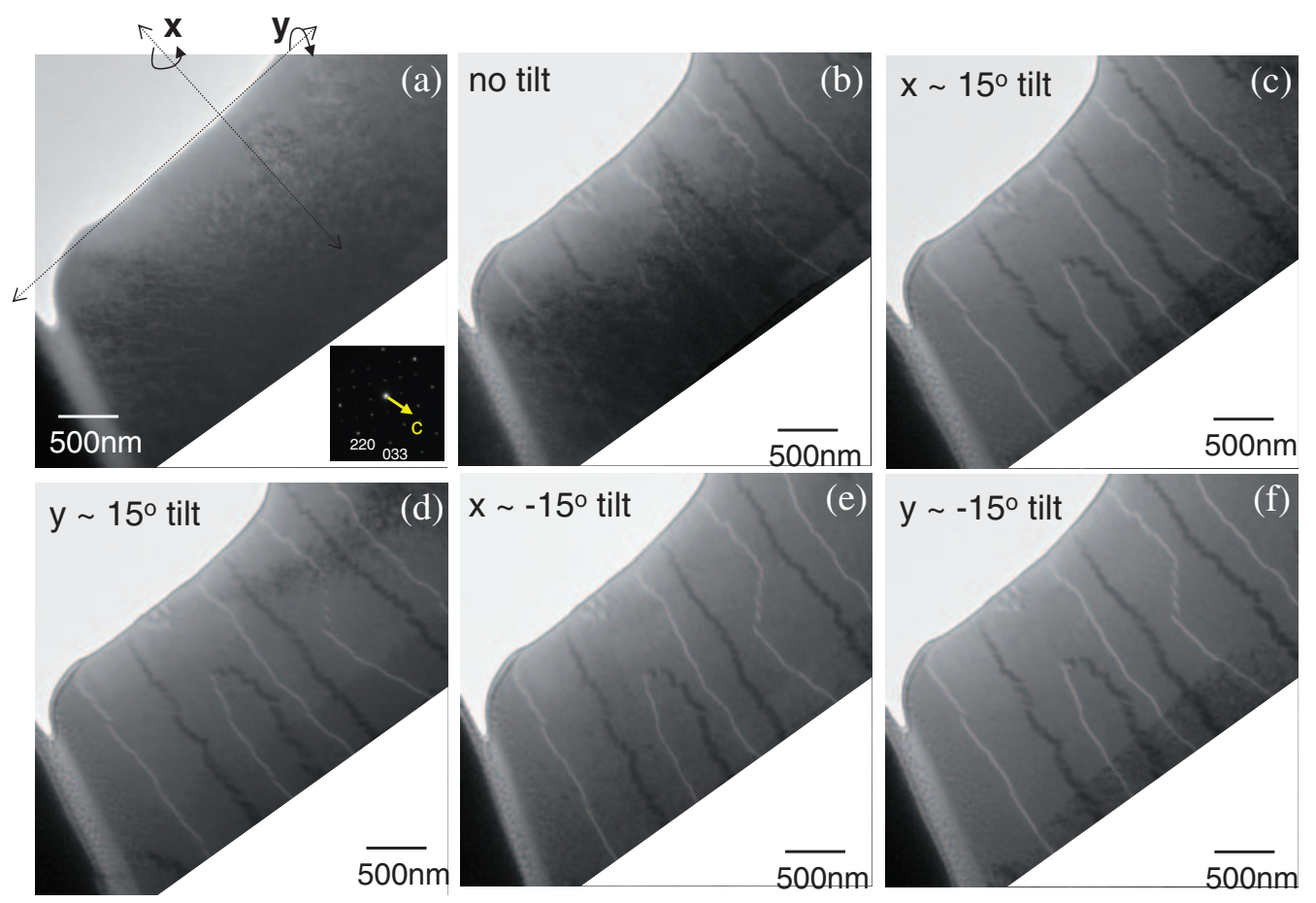

Fig. 3 Lorentz microscope images of the $\mathrm{Sm}_{2}\left(\mathrm{Fe}_{0.95}, \mathrm{Mn}_{0.05}\right)_{17} \mathrm{~N}_{5.0}$ powder obtained when the specimen is tilted. The magnetization easy axis is indicated by a yellow arrow. (a) Just-focused image and diffraction pattern (inset). (b)-(f) Corresponding underfocused images. The tilting angles are indicated in the top-left corners. 

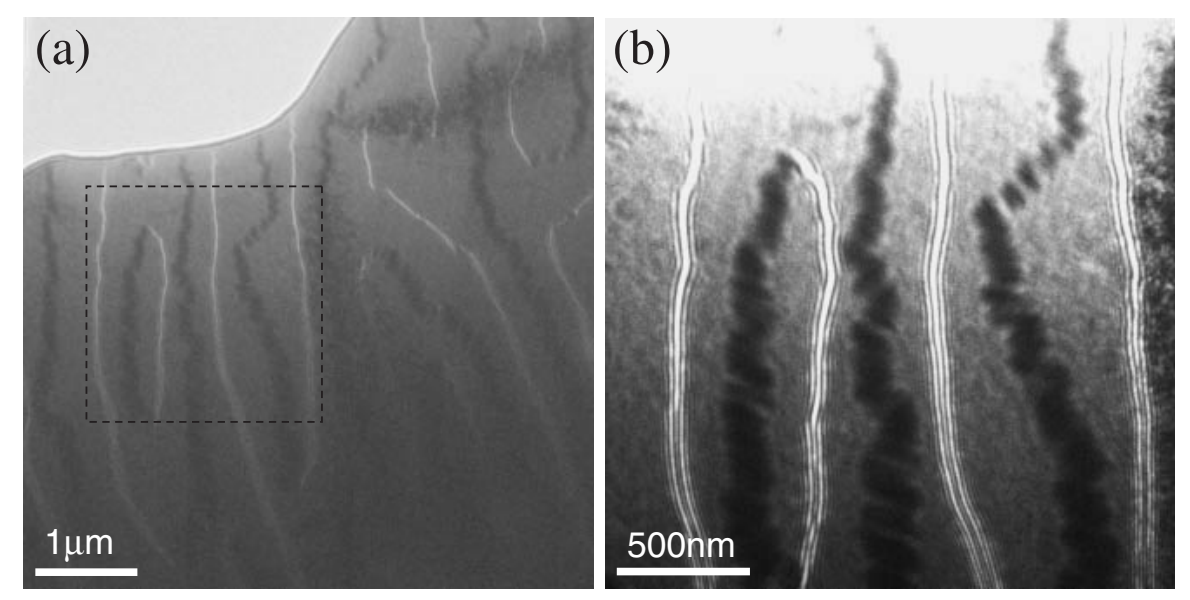

Fig. 4 Lorentz microscope images taken in the severely underfocused condition. (b) Enlarged image obtained for the rectangle indicated by black broken lines in (a).
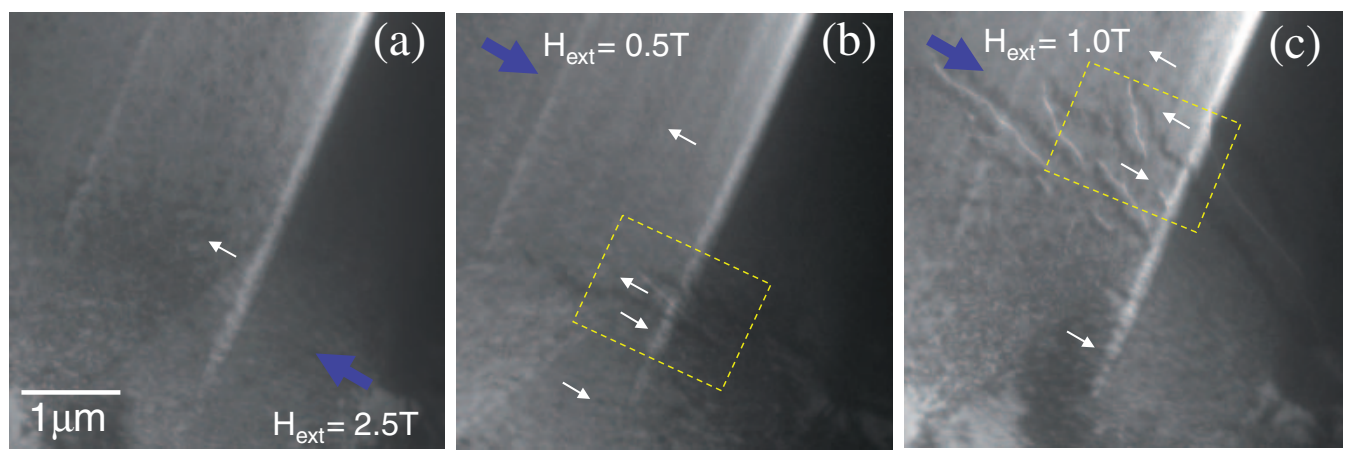

Fig. 5 Lorentz microscope images showing the magnetization process in the $\mathrm{Sm}_{2}\left(\mathrm{Fe}_{0.95}, \mathrm{Mn}_{0.05}\right){ }_{17} \mathrm{~N}_{5.0}$ powder. The blue arrows indicate the direction of the applied external magnetic field. Note that the external magnetic field is applied along the direction parallel to the magnetization easy axis. The white arrows indicate the magnetization direction.

On the other hand, by means of ex situ observations conducted using electron holography in combination with Lorentz microscopy, we can again confirm that the amorphous layer acts as a pinning center. Figure 7 shows the Lorentz microscope images (left) and reconstructed phase images (right) of the $\mathrm{Sm}_{2}\left(\mathrm{Fe}_{0.95}, \mathrm{Mn}_{0.05}\right)_{17} \mathrm{~N}_{5.0}$ powder that is subjected to a magnetic field generated using an electromagnet. The reconstructed phase images are obtained for the black rectangular region of the Lorentz microscope image shown in Fig. 7(a). In the reconstructed phase image, the direction and density of the white lines correspond to those of the lines of magnetic flux projected along the electron beam. ${ }^{12)}$ In the demagnetized state shown in Fig. 7(a), white and black lines can be observed in the region where the directions of the lines of magnetic flux tend to change, as indicated by the yellow lines. Thus, it is considered that in the region where the directions of the lines of magnetic flux change, the white and black lines observed by Lorentz microscopy correspond to the domain walls. When a magnetic field of $1.0 \mathrm{~T}$ is applied to the specimen, some domain walls undergo slight changes, as shown in Fig. 7(b). It is found that in Fig. 7(c), the domain walls completely disappear. On the other hand, in the reconstructed phase image shown in Fig. 7(c), the lines of magnetic flux on the left/center sides of the image are not aligned along the applied magnetic field, while the lines of magnetic flux on the right side are aligned. It is noted that on the right side of Fig. 7(c), a considerable change in the thickness of the specimen occurs due to the use of the FIB milling process. Thus, it is considered that the stray fields observed on the left/center sides of the reconstructed phase image shown in Fig. 7(c) are affected by the thick region of the specimen. When a magnetic field of $1.0 \mathrm{~T}$ is oppositely applied to the specimen, the domain walls are again observed, as shown in Fig. 7(d). Here, we note two phenomena. First, the domain walls are located in the amorphous layers after domain wall motion; in the Lorentz microscope image shown in Fig. 7(c) (left), we indicate the amorphous layers by numbered blue arrowheads, which correspond to the domain wall indicated by yellow lines in the results of the reconstructed phase images (right). Second, the position of the domain wall in the green square shown in Fig. 7(d) is almost the same as that of the domain wall in Fig. 7(b). These results indicate that the coercivity of the $\mathrm{Sm}_{2}\left(\mathrm{Fe}_{0.95}, \mathrm{Mn}_{0.05}\right)_{17} \mathrm{~N}_{5.0}$ powder is strongly affected by the distribution of amorphous layers.

\section{Conclusions}

The magnetic domain structure and magnetization process in $\mathrm{Sm}_{2}\left(\mathrm{Fe}_{0.95}, \mathrm{Mn}_{0.05}\right)_{17} \mathrm{~N}_{5}$ powders are investigated by Lorentz microscopy and electron holography. The results are summarized as follows: 

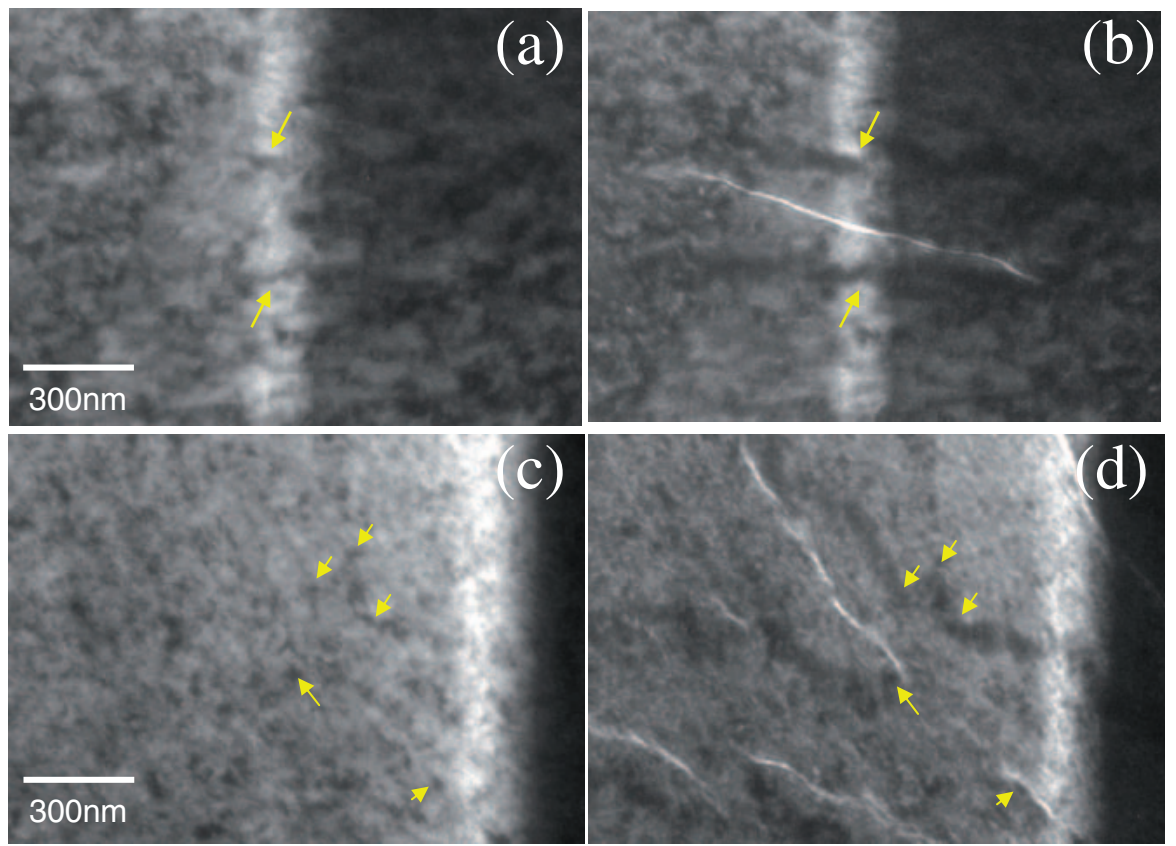

Fig. $6(\mathrm{a}, \mathrm{b})$ and $(\mathrm{c}, \mathrm{d})$ are the enlarged Lorentz microscope images obtained for the rectangles indicated by the yellow lines shown in Figs. 5(b) and (c), respectively.

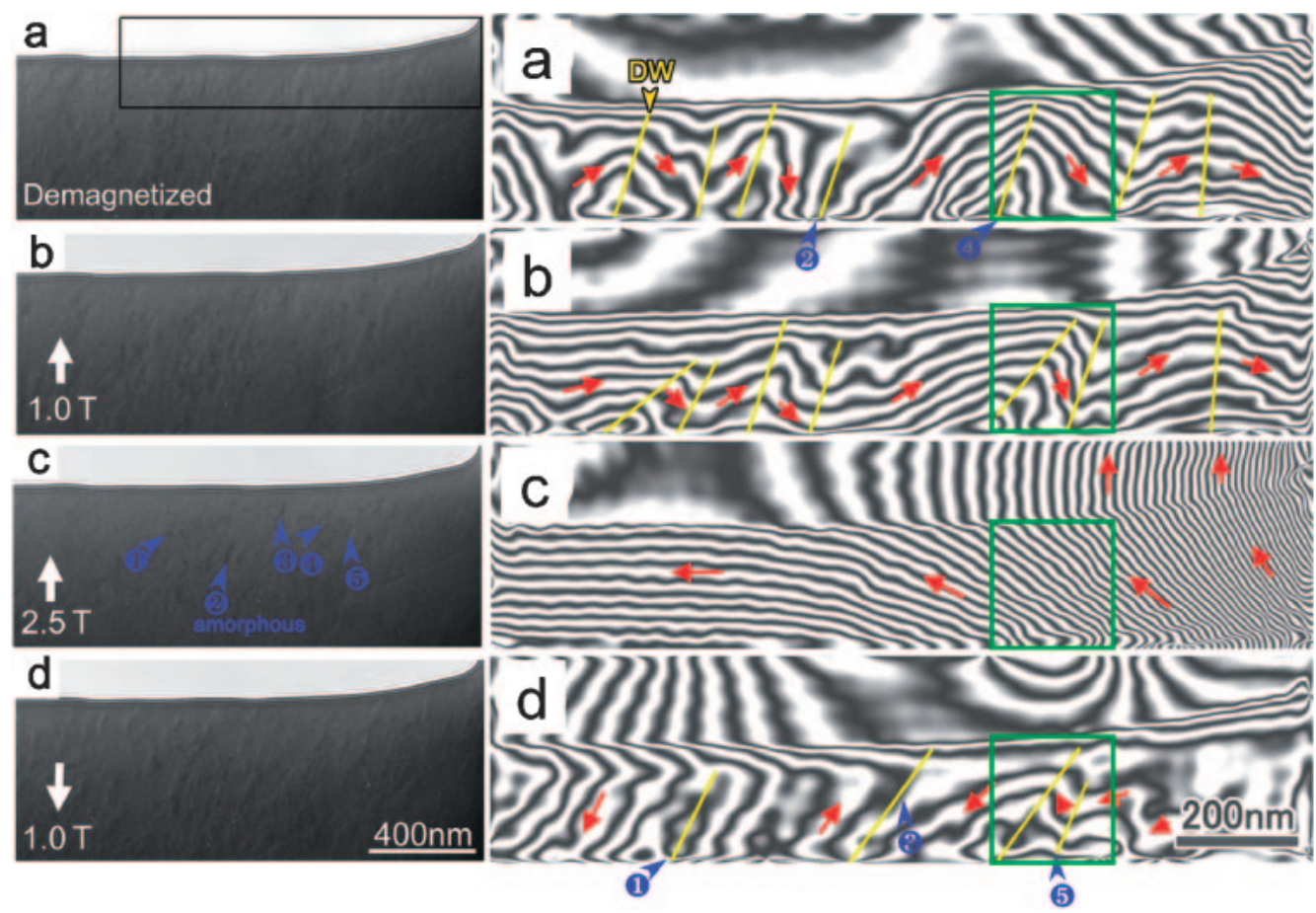

Fig. 7 Lorentz microscope images (left) and reconstructed phase images (right) of the $\mathrm{Sm}_{2}\left(\mathrm{Fe}_{0.95}, \mathrm{Mn}_{0.05}\right)_{17} \mathrm{~N}_{5.0}$ powder subjected to a magnetic field generated by an electromagnet. DW (yellow lines) indicates the domain wall. The white and red arrows indicate the direction of the applied magnetic field and the directions of the lines of magnetic flux, respectively.

(1) The magnetic domain walls in the $\mathrm{Sm}_{2}\left(\mathrm{Fe}_{0.95}\right.$, $\left.\mathrm{Mn}_{0.05}\right)_{17} \mathrm{~N}_{5}$ powder are aligned along the direction parallel to the magnetization easy axis (c axis). They are located in the amorphous layers distributed along the direction parallel to the c axis.

(2) The domain walls in the thin foil specimen, which are connected with one another along the amorphous layers, exist in the region from the top surface to the bottom surface of the specimen having a thickness of approximately $100 \mathrm{~nm}$.

(3) From the ex situ observations of the magnetization process, it is found that the domain walls are always located in the amorphous layers even after domain wall motion. This result reveals that the amorphous layer 
acts as an attractive pinning center for domain wall motion, resulting in the large coercivity $(1.04 \mathrm{MA} / \mathrm{m})$ of the $\mathrm{Sm}_{2}\left(\mathrm{Fe}_{0.95}, \mathrm{Mn}_{0.05}\right){ }_{17} \mathrm{~N}_{5.0}$ powder.

\section{Acknowledgments}

This work was partly supported by a Grant-in-Aid for Scientific Research (A) from the Japan Society for the Promotion of Science.

\section{REFERENCES}

1) T. Iriyama, et al.: IEEE Trans. Magn. 28 (1992) 2326-2331.

2) S. Suzuki, T. Miura and M. Kawasaki: IEEE Trans. Magn. 29 (1993)
2815-2817.

3) K. Majima, M. Ito, S. Katsuyama and H. Nagai: J. Appl. Phys. 81 (1997) 4530-4532.

4) N. Imaoka, et al.: J. Alloys Compd. 222 (1995) 73-77.

5) K. Zuzek, P. J. McGuiness and S. Kobe: J. Alloys Compd. 289 (1999) 265-269.

6) R. Arlot, et al.: Int. J. Hydrogen Energy 24 (1999) 661-664

7) W. Kaszuwara, M. Leonowicz and J. A. Kozubowsk: Mater. Lett. 42 (2000) 383-386.

8) T. Iseki, T. Ishikawa, A. Kawamoto and K. Ohmori: J. Jpn. Soc. Powder Powder Metallurgy. 50 (2003) 633-637.

9) X. C. Kou, W. J. Qiang and H. Kromüller: J. Appl. Phys. 74 (1993) 6791-6797.

10) A. Yasuhara, et al.: J. Magn. Magn. Mater. 295 (2005) 1-6.

11) D. Shindo, Y.-G. Park, Y. Murakami, Y. Gao, H. Kanekiyo and S. Hirosawa: Scripta Mater. 48 (2003) 851-856.

12) D. Shindo and T. Oikawa: Analytical Electron Microscopy for Materials Science (Springer, 2002) 111-125. 